\title{
Triptolide induces apoptosis in endometrial cancer via a p53-independent mitochondrial pathway
}

\author{
XIAO-FEI WANG ${ }^{1}$, YI-BING ZHAO ${ }^{2}$, QIANG WU², ZHI-HUA SUN ${ }^{2}$ and HAI-JIN LI ${ }^{2}$ \\ ${ }^{1}$ Changzhou Maternal and Child Health Care Hospital of Nanjing Medical University, Changzhou 213003; \\ ${ }^{2}$ Department of Gynecological Oncology, Jiangsu Cancer Hospital of Nanjing Medical University, Nanjing 210009, P.R. China
}

Received October 8, 2012; Accepted January 18, 2013

DOI: $10.3892 / \mathrm{mmr} .2013 .1783$

\begin{abstract}
Triptolide (TP), the primary active component purified from the traditional Chinese herbal medicine Tripterygium wilfordii Hook. F (TWHF), has been shown to possess antitumor activity in several types of solid tumors. In the present study, we investigated the antitumor effect of TP in human endometrial cancer cells (HEC-1B) and elucidated its possible underlying mechanisms. HEC-1B cells were treated with various doses of TP $(10,20,40,80$, 160 and $320 \mathrm{nM}$ ), and the cell viability was assessed by Cell Counting Kit-8 (CCK-8) and flow cytometric analysis. Results indicated that TP inhibited the proliferation of HEC-1B cells in a dose- and time-dependent manner. To further investigate its mechanisms, the levels of apoptosis and the changes in caspase-3/9 expression in HEC-1B cells by pretreatment with z-VAD-fmk, a pan-caspase inhibitor, were detected by CCK-8 and western blotting. The cytotoxic effects of TP were significantly inhibited by z-VAD-fmk. At the molecular level, TP did not effectively activate the p53 signaling pathway, but upregulated caspase-3/9 and downregulated bcl-2 without changing the bax level. Our studies revealed that TP has an effect on the apoptotic ability of endometrial cancer cells via a p53-independent mitochondrial pathway, presenting a novel strategy to evade drug resistance in tumorigenesis. The ability of TP to be a potential chemotherapeutic agent for endometrial cancer should be considered.
\end{abstract}

\section{Introduction}

Endometrial cancer is the most common invasive malignant neoplasm of the female genital system, with an estimated 46,470 diagnosed cases and 8,120 deaths in 2011 in the United States (1). The treatment of endometrial cancer involves

Correspondence to: Dr Qiang Wu or Dr Zhi-Hua Sun, Department of Gynecological Oncology, Jiangsu Cancer Hospital of Nanjing Medical University, 42 Baiziting Road, Nanjing 210009, P.R. China E-mail: qiangw88@126.com

E-mail:szhnj@163.com

Key words: triptolide, endometrial cancer, mitochondrial pathway, caspase, bcl-2 family, p53 pathway surgery combined with radiotherapy, chemotherapy and biological treatment (2). Previous studies demonstrate high loco-regional control in early-stage endometrial cancer treated with adjuvant pelvic external radiotherapy. However, overall survival remains largely unaffected (3). For patients with advanced stage disease or recurrent disease, particularly when it is not amenable to surgical resection, systemic chemotherapy is often required as adjuvant treatment (4). Therefore, it is beneficial to develop a novel therapeutic agent for patients at risk of micrometastatic disease.

Currently, more and more attention is being paid to phytochemicals and herbal extracts due to their inhibitory abilities against cancer cell growth and metastasis, which are suggested to improve the anticancer effect of standard treatments as novel chemotherapeutic agents or adjuvants (5). Triptolide (TP), a highly oxygenated diterpene isolated from Tripterygium wilfordii Hook. F, one of the main active components in TWHF, has been used in traditional Chinese medicine for centuries. It has been demonstrated to be effective in patients with a variety of inflammatory and autoimmune diseases (6). In recent studies, TP has also been shown to have effective antiproliferative activity and to induce apoptosis in a broad range of types of cancer in vitro and in vivo, including colon, breast, prostate, urothelial and hematological cancer (7-11). However, the effect of TP on human endometrial cancer has not yet been well described. Thus, we attempted to identify the mechanism of TP-induced apoptosis in human endometrial cancer cells (HEC-1B) in vitro. Previous studies from our group demonstrated that TP was able to induce prominent growth inhibition and apoptosis in HEC-1B cells (12-14).

This study aimed to investigate the exact targets and molecular mechanisms of the pro-apoptosis action of TP on HEC-1B cells. It was reported that TP was capable of inducing apoptosis of HEC-1B cells via the p53-independent pathway, but not the mitochondrial-mediated pathway. Our results suggested that TP may be a useful anticancer agent in endometrial cancer.

\section{Materials and methods}

Cell culture and drugs. The human endometrial cancer cells (HEC-1B) were obtained from the Chinese Academy of Sciences Shanghai cell bank. The cell lines were cultured in DMEM (Gibco BRL, Carlsbad, CA, USA), fetal bovine serum (FBS; Gibco BRL), $100 \mathrm{U} / \mathrm{ml}$ penicillin and $100 \mu \mathrm{g} / \mathrm{ml}$ 
streptomycin in a humidified atmosphere containing $5 \% \mathrm{CO}_{2}$ at $37^{\circ} \mathrm{C}$. TP was obtained from National Institutes for Food and Drug Control and dissolved in DMSO (Sigma, St. Louis, MO, USA). The pan-caspase inhibitor z-VAD-fmk was obtained from Beyotime (Jiangsu, China).

Cell proliferation assay. Cells were seeded in a 96-well dish at a density of $4 \times 10^{3}$ cells per well and incubated in DMEM containing 10\% FBS for $24 \mathrm{~h}$. After exposure to the drug for 24, 48 and $72 \mathrm{~h}$, the cells were washed with PBS and incubated in $100 \mu$ l DMEM containing $10 \mu \mathrm{l}$ Cell Counting Kit-8 (CCK-8; Dojindo, Japan) solution for $90 \mathrm{~min}$. The absorbance of each well was measured at a wavelength of $450 \mathrm{~nm}$. Five duplicate wells were used for each measurement and experiments were repeated three times.

Annexin V/PI staining assay. Following treatment with $\mathrm{TP}$ for $48 \mathrm{~h}, \sim 1 \times 10^{5}$ cells $/ \mathrm{ml}$ cells were harvested by $0.5 \%$ Trypsin-EDTA (Gibco BRL), washed twice with cold PBS and resuspended in $200 \mu \mathrm{l}$ binding buffer. Cell suspension was added to $5 \mu \mathrm{l}$ Annexin V-FITC (Becton Dickinson, Bedford, MA, USA) and $5 \mu \mathrm{l}$ propidium iodide (PI; Becton Dickinson), incubated for $15 \mathrm{~min}$ at room temperature in the dark according to the manufacturer's instructions and the percentage of apoptotic cells in a 10,000-cell cohort was analyzed by a FACSCalibur flow cytometer (Becton Dickinson). The early and late apoptotic/necrotic cells were determined as the percentage of Annexin $\mathrm{V}^{+} / \mathrm{PI}^{-}$and Annexin $\mathrm{V}^{+} / \mathrm{PI}^{+}$cells.

Hoechst 33342 staining. The apoptotic effect of TP on HEC-1B cells was analyzed by DNA staining. HEC-1B cells were placed in the wells of a 24-well plate. After $24 \mathrm{~h}$ cell culture, the cells were treated with TP for $48 \mathrm{~h}$. The cells were washed with PBS three times and fixed in $4 \%$ paraformaldehyde for $10 \mathrm{~min}$ and stained with Hoechst 33342 at a concentration of $5 \mu \mathrm{g} / \mathrm{ml}$ (Beyotime) in the dark at room temperature for $30 \mathrm{~min}$. After washing with PBS twice, the fluorescent chromosomal DNA morphology was observed by fluorescence microscopy with a 340-nm excitation filter. Apoptotic cells were identified by morphology and condensation of the nuclei.

Real-time PCR. Total RNA was isolated from cells using TRIzol reagent (Invitrogen, Carlsbad, CA, USA) after treatment with TP for $48 \mathrm{~h}$. High-quality RNA, having a $28 \mathrm{~S} / 18 \mathrm{~S}$ rRNA ratio of 1.5:2 was used for further experimentation. Equal quantities of RNA (1000 ng) from each sample were used as templates for cDNA synthesis with the PrimeScript RT Master Mix (Takara Biotechnology, Dalian, China) according to the manufacturer's instructions. Real-time PCR assays were carried out using Power SYBR Green PCR Master Mix kit (Applied Biosystems, Carlsbad, CA, USA) in a total volume of $20 \mu \mathrm{l}$ on the ABI Prism 7300 Sequence Detection System (Applied Biosystems). The primer sequences for bcl-2: forward, 5'-CGGTTCAGGTACTCAGTCATCC-3'; reverse, 5'-GGTGGGGTCATGTGTGTGG-3'; bax: forward, 5'-CCCGAGAGGTCTTTTTCCGAG-3'; reverse, 5'-CCAGCCCATGATGGTTCTGAT-3'; $\beta$-actin: forward, 5'-TTCTACAATGAGCTGCGTGTG-3'; reverse, 5'-CAGCCTGGATAGCAACGTACA-3'. The reaction parameters of bcl-2, bax and $\beta$-actin were as follows: one cycle at
A

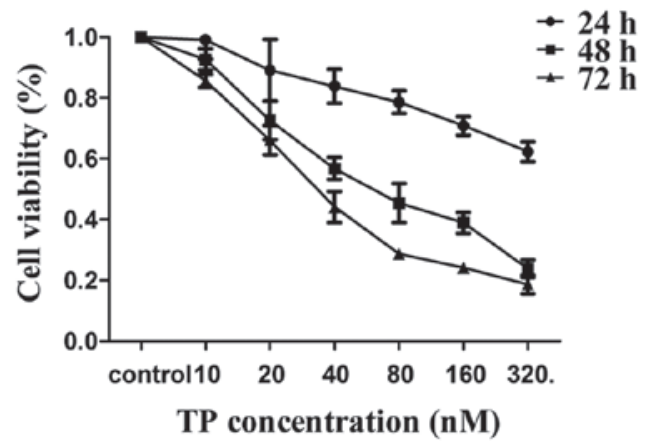

B

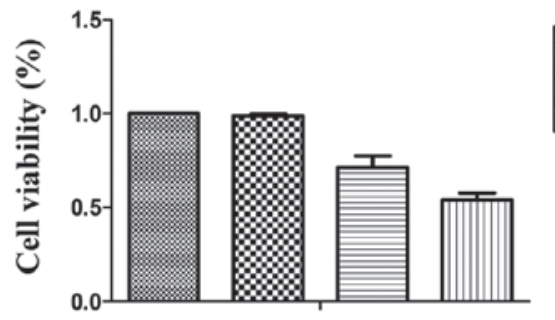

Figure 1. Effect of triptolide (TP) on the growth inhibition of HEC-1B cell lines. (A) Exponentially growing cell lines were maintained in DMEM with $10 \%$ fetal bovine serum for $24 \mathrm{~h}$, and then treated for 24,48 and $72 \mathrm{~h}$ with the indicated concentration of TP. Cell growth inhibition was assessed by CCK-8 assay. (B) HEC-1B cells were pretreated with $20 \mu \mathrm{M} \mathrm{z}$-VAD-fmk for $1 \mathrm{~h}$, followed by $40 \mathrm{nM}$ TP for $48 \mathrm{~h}$, and the apoptosis of cells were determined by CCK- 8 assay. Data are the means \pm SD of three independent experiments.

$95^{\circ} \mathrm{C}$ for $30 \mathrm{sec}$ and 40 cycles of denaturation at $95^{\circ} \mathrm{C}$ for $5 \mathrm{sec}$, annealing at $60^{\circ} \mathrm{C}$ for $30 \mathrm{sec}$. Standard curves were prepared for target and reference ( $\beta$-actin) genes.

Western blot analysis. Cells were treated with various concentrations of TP for the indicated time in DMEM with 10\% FBS. The cells were collected in ice-cold PBS and prepared in RIPA buffer with PMSF (Beyotime) for $30 \mathrm{~min}$ at $4^{\circ} \mathrm{C}$. The protein concentrations of the cell lysates were boiled with gel-loading buffer at $100^{\circ} \mathrm{C}$ for $5 \mathrm{~min}$. An equal amount of protein was electrophoresed on 10-15\% SDS-polyacrylamide gels and transferred onto PVDF membranes (Millipore, Billerica, MA, USA). Membranes were blocked with $5 \%$ nonfat milk in Trisbuffered saline with $0.1 \%$ Tween-20 (TBST) for $2 \mathrm{~h}$ at room temperature and then incubated with the appropriate primary antibodies including caspase-3/9 (Cell Signaling, Danvers, MA, USA), p53 (Bioworld, St. Louis, MO, USA) and bcl-2/ bax (Santa Cruz Biotechnology Inc., Santa Cruz, CA, USA) in PBST overnight at $4^{\circ} \mathrm{C}$. After washing three times in PBST, the membranes were incubated with horseradish peroxidaseconjugated appropriate secondary antibodies for $90 \mathrm{~min}$ at room temperature. The blots were revealed by enhanced chemiluminescene (ECL; Millipore) according to the manufacturer's instructions.

Statistical analysis. Values are expressed as the means \pm SD. Data were analyzed by a one-way analysis of variance (ANOVA). The difference was considered statistically significant if $\mathrm{P}<0.05$. All analyses were performed using GraphPad Prism 5 software version. All statistical tests were three-sided. 
A

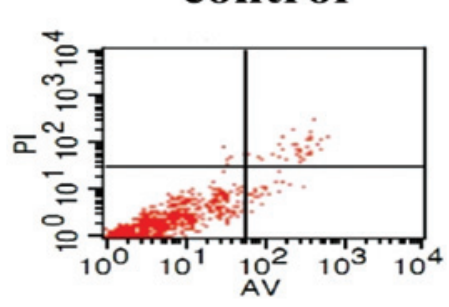

$40 \mathrm{nM}$
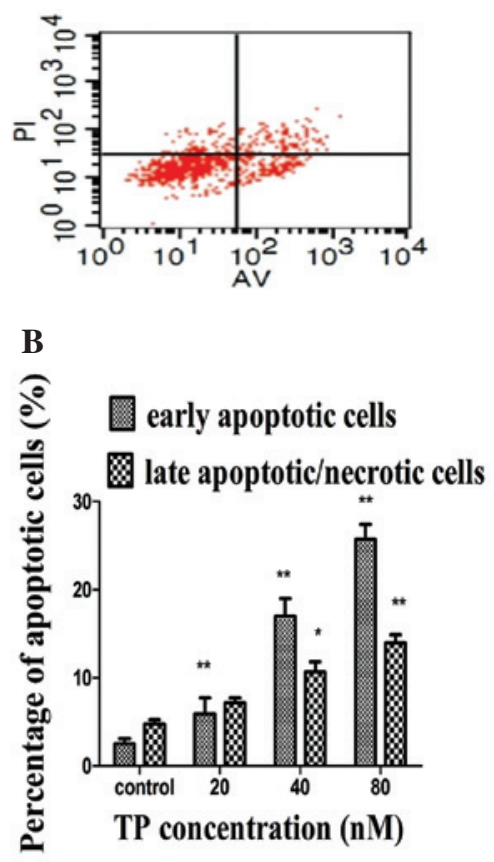

\section{$20 \mathrm{nM}$}

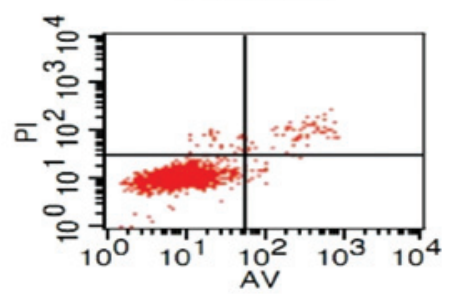

80 nM

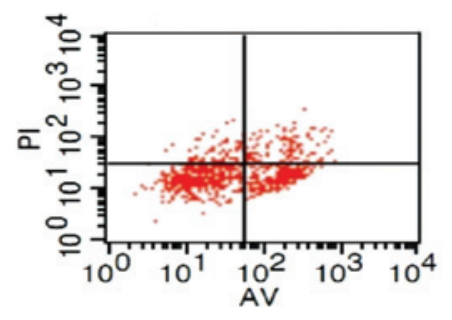

C

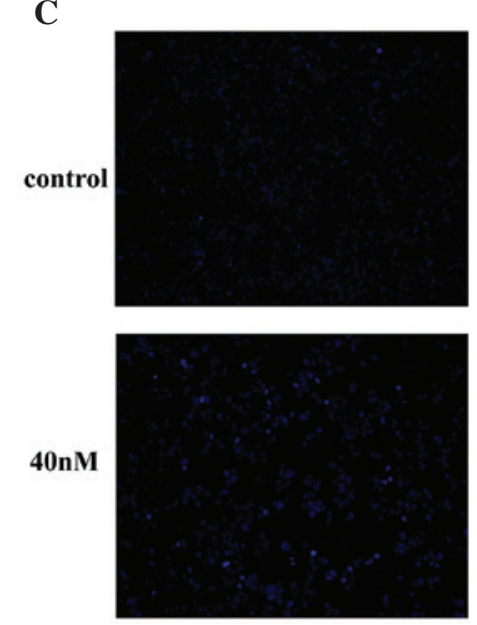

Figure 2. TP induced apoptosis of HEC-1B cells. (A) After treatment with the indicated concentrations of TP (nM) for 48 h, cells were stained with Annexin V and PI. (B) Percentages of early apoptotic (Annexin $\mathrm{V}^{+}$and $\mathrm{PI}^{-}$) and late apoptotic/necrotic (Annexin $\mathrm{V}^{+}$and $\mathrm{PI}^{+}$) cells are shown by a flow cytometry analysis ${ }^{*} \mathrm{P}<0.05,{ }^{* * *} \mathrm{P}<0.01$ compared with the control. Data are reported as the means \pm SD of three independent experiments.(C) Hoechst 33342 staining showing death of HEC-1B cells following exposure to $40 \mathrm{nM}$ TP for $48 \mathrm{~h}$ (magnification, $\mathrm{x} 400$ ). TP, triptolide; PI, propidium iodide.

\section{Results}

Effect of TP on the growth of HEC-1B cell lines. In our current study, we examined the effect of TP on the human endometrial cancer cell line HEC-1B by CCK-8 assay. Cells were treated with various concentrations of TP for 24,48 and 72 h. Fig. 1A shows the dose-dependent effect of TP after time-dependent exposure. Following $48 \mathrm{~h}$ of treatment, the majority of HEC-1B cell lines were reduced by TP therapy, indicating the existence of cell death induced by TP. To further question the role of caspase activation in TP-induced apoptosis, we treated HEC-1B cells with the pan-caspase inhibitor z-VAD-fmk $(20 \mu \mathrm{M})$ prior to TP treatment. Pretreatment with the pancaspase inhibitor z-VAD-fmk reduced TP-induced apoptosis (Fig. 1B).

TP induces apoptosis in HEC-1B cells. To understand the mechanisms of TP-induced cell death in endometrial cancer cells, we examined the ability of TP to induce apoptosis using Annexin V/PI by flow cytometry analyses. As shown in
Fig. 2A, $80 \mathrm{nM}$ TP for $48 \mathrm{~h}$ resulted in apoptosis in a large percentage of cells. This assay divided apoptotic cells into early and late apoptosis/necrosis. We observed that treatment with various concentrations of TP for $48 \mathrm{~h}$ resulted in a concentration-dependent increase in both early and late apoptotic/necrotic cells. The percentage of early apoptotic cells was larger than that of late apoptotic/necrotic cells (Fig. 2B). Hoechst 33342 staining revealed that there were significant morphological changes in the nuclear chromatin of the HEC-1B cells after treatment with $40 \mathrm{nM}$ TP for $48 \mathrm{~h}$. Compared with the normal morphological nuclei in the control group, condensed chromatin or fragmented nuclei were observed in the treated cells (Fig. 2C).

$T P$ regulates the expression of bcl-2 family and $p 53$ proteins in HEC-1B cells. We tested the effects of TP on the expression of the anti-apoptotic protein bcl-2 and the pro-apoptotic protein bax using real-time PCR in HEC-1B cells. As shown in Fig. 3A, TP reduced the mRNA levels of bcl-2 in a dose-dependent manner, while no significant changes in the 
A

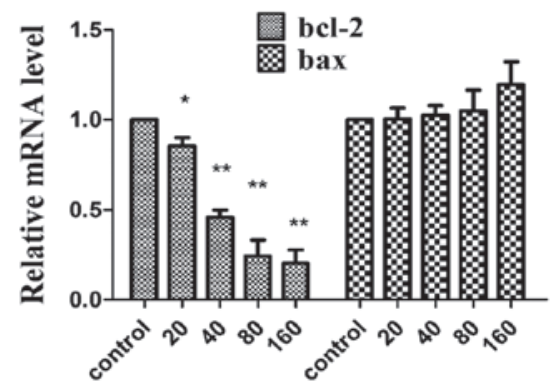

TP concentration (nM)

B

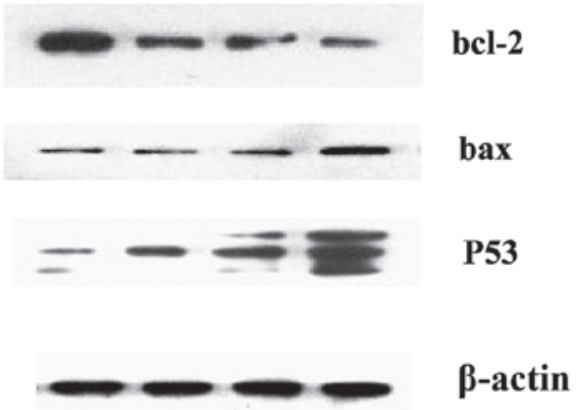

Figure 3. TP regulates the expression of bcl-2 family and $\mathrm{p} 53$ proteins in HEC-1B cell lines. (A) HEC-1B cell lines were treated with the indicated concentrations of TP for $48 \mathrm{~h}$. The mRNA levels of bcl-2 and bax were determined by real-time PCR and normalized to $\beta$-actin. Data are the means \pm SD of three separate experiments; ${ }^{*} \mathrm{P}<0.05,{ }^{* *} \mathrm{P}<0.01$ compared with the control. (B) Cells were treated with the indicated concentrations of TP $(0,20,40$, $80 \mathrm{nM}$ ) for $48 \mathrm{~h}$, and total cellular extracts were prepared and subjected to western blot assay using the antibodies anti-bcl-2, bax, p53 and $\beta$-actin. $\beta$-actin served as a loading control.

A

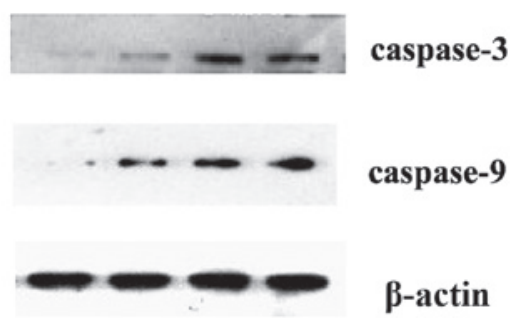

B

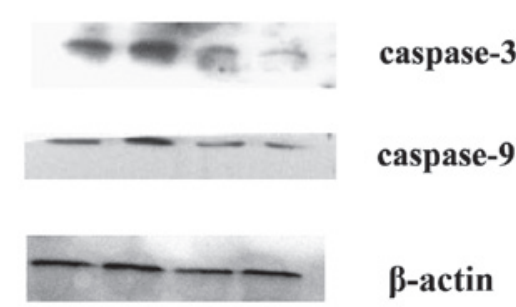

Figure 4. Effect of TP on the expression of caspase-3 and caspase-9. (A) HEC-1B cells were treated with the indicated concentrations of TP $(0$, $20,40,80 \mathrm{nM}$ ) for $48 \mathrm{~h}$, and western blot analysis was used to investigate the expression levels of caspase-3 and caspase-9. (B) Cells were pretreated with or without z-VAD-fmk $(20 \mu \mathrm{M})$ for $1 \mathrm{~h}$, followed with TP $(40 \mathrm{nM})$ for $48 \mathrm{~h}$. Western blots show the effect of $\mathrm{z}-\mathrm{VAD}$-fmk on the expression of caspase-3 and caspase-9. Protien levels of $\beta$-actin were measured as a control.

expression levels of bax were observed. Furthermore, similar results were observed using western blotting: the protein expression of bcl-2 was reduced, whereas bax protein expres- sion remained mostly constant with no significant changes. Bax protein was slightly reduced in $80 \mathrm{nM}$ TP, accompanied by a marked enhanced p53 protein expression (Fig. 3B).

Expression of caspase- 3 and caspase-9 proteins. Time- and dose-dependent effects of TP on the expression of caspase-3 and caspase- 9 were examined in our study. As shown in Fig. 4A, TP treatment resulted in significantly increased expression of caspase-3 and caspase-9 in HEC-1B cells. Furthermore, to assess the role of caspase-3/9 in TP-induced apoptosis in HEC-1B cells, we pretreated HEC-1B cells with the pan-caspase inhibitor z-VAD-fmk $(20 \mu \mathrm{M})$. The protein levels of caspase- 3 and caspase- 9 were reduced by pretreatment with pan-caspase inhibitor z-VAD-fmk, although they were still higher than the control (Fig. 4B).

\section{Discussion}

In our present study, we demonstrated that TP, a compound purified from a traditional Chinese herb, induced effective growth inhibition and cell apoptosis in human endometrial cancer HEC-1B cells in a dose- and time-dependent manner. Furthermore, the Annexin V/PI staining assay demonstrated that TP induced apoptosis, but not necrosis, in HEC-1B cells. Apoptotic cells lose asymmetry of membrane phospholipids, which induces the phosphatidylserine (PS) translocation from the inner leaflet of the plasma membrane to the outer layer (PS also appears on the surface of necrotic cells), and can bind with Annexin V (15). PI is a standard flow cytometric viability probe for staining the cellular DNA of late apoptotic/necrotic cells with compromised cell membranes, whereas it excluded normal or early apoptotic cells with intact membranes (16). It is considered that viable cells are Annexin $\mathrm{V}^{-}$and $\mathrm{PI}^{-}$, early apoptotic cells are Annexin $\mathrm{V}^{+}$and $\mathrm{PI}^{-}$, and late apoptotic/ necrotic cells are Annexin $\mathrm{V}^{+}$and $\mathrm{PI}^{+}$. Annexin $\mathrm{V}$ may be used in combination with PI to detect apoptotic cells and discriminate between apoptosis and necrosis (17). In the present study, the Annexin V/PI assay showed that TP treatment increased the proportion of early apoptotic HEC-1B cells. Apoptosis is characterized by chromatin condensation, which was observed by Hoechst 33342 staining in HEC-1B cells with TP treatment at a concentration of $40 \mathrm{nM}$. We showed that TP markedly and significantly increased the number of apoptotic cells in endometrial cancer cell lines, which suggested that TP may be useful as an effective therapeutic reagent for endometrial cancer.

TP has been shown to promote apoptosis by inducing mitochondrial damage and decreasing the expression of antiapoptotic proteins in a number of types of tumor cells (18). This is the first study to demonstrate involvement of the mitochondrial pathway in TP-induced apoptosis in human endometrial cancer HEC-1B cell lines. There are two major intracellular apoptosis signaling pathways, the mitochondrial pathway and the death receptor pathway. The mitochondrial pathway is regulated by members of the bcl-2 protein family, which can be subdivided into anti-apoptotic proteins, such as bcl-2 and bcl-xl, and pro-apoptotic proteins, such as bax and bad (19). The bcl-2 family is an important group of proteins that was first found to alter the permeability of the outer mitochondrial membrane to regulate the release of apoptotic proteins from 
the mitochondria (20). Bcl-2 is the first protein involved in the regulation of apoptosis to be molecularly characterized. It does not rely on cell division to prevent programmed cell apoptosis. Bax translocation from the cytosol to the mitochondria induces cytochrome c release, whereas overexpression of the anti-apoptotic protein bcl-2 may inhibit the activity of the pro-apoptotic protein bax by inhibiting the translocation of bax to the mitochondria and causing aberrant proliferation and abnormal cell survival $(16,21)$. Clearly, the ratio of bcl-2/bax expression is crucial for controlling cell apoptosis (22). In our study, the protein level of bcl-2 was significantly decreased, while the protein level of bax was unchanged by real-time PCR and western blotting. The alteration of the bcl-2/bax ratio may be in favor of apoptosis in HEC-1B cells.

A key event in apoptosis is the release of mitochondrial intermembrane space proteins to the cytosol, such as cytochrome c. Furthermore, the release of cytochrome $\mathrm{c}$ from the mitochondria into the cytosol to activate caspase frequently results in activation of the mitochondrial pathway (23). Among the variety of cellular proteins that play a role in the induction of apoptosis, caspases are required for the execution of apoptotic stimuli, inducing cell death $(24,25)$. We observed that TP exposure potently induced apoptosis through increasing the expression of caspase-3/9, which was associated with reduced bcl-2. Furthermore, the caspase inhibitor z-VAD-fmk blocked TP-induced cell death, indicating that TP-induced cell death in HEC-1B cells is caspase-dependent. A mitochondrial breach is often triggered by caspases rather than being required for caspase activation (26). Therefore, the mitochondrial apoptosis pathway plays a critical role in TP-induced cell death, in accordance with other cancer cell lines undergoing TP treatment (27).

There are several survival mechanisms that enable cancer cells to escape the cell death induced by chemotherapeutic drugs. Mutant p53 is one of particular significance (28). As p53-mediated pathways are used to induce growth arrest or apoptosis by a number of chemotherapeutic drugs, it has been suggested that dysfunction of p53 may partially explain the resistance of endometrial cancer to drug treatment. Previous studies have shown that TP promotes the apoptosis of tumor cells via the p53-dependent pathway $(10,29)$ or p53-independent pathway $(30,31)$. In human tumors, the reactivation of p53 to induce apoptosis of abnormal cells depends on the downstream pro-apoptotic protein bax (32). In the present study, we found that TP was capable of upregulating the level of p53 without changing the expression of bax, indicating that the p53 pathway was not effectively activated. We therefore hypothesize that TP using p53-independent pathways may be most efficacious against endometrial cancer. In addition, the expression of bcl-2 by tumors may induce a variety of antitumor agents to protect cancer cells from programmed cell death in order to confer resistance to chemotherapy (33). Our results showed that TP led to increased levels of p53 with downregulation of the expression of bcl-2, while there was no change in the level of bax expression, indicating that TP is an effective novel therapeutic agent to resolve the aberrant tumor growth and drug resistance in human endometrial cancer.

In conclusion, our data revealed that $\mathrm{TP}$ induces human endometrial cancer HEC-1B cell apoptosis via the mitochondrial pathway, not the p53-dependent pathway. The current study demonstrated that TP was effective as a single agent in inducing apoptosis of human endometrial cancer cell lines and further suggested the potential of TP combining with other chemotherapeutic agents or radiotherapy for the treatment of advanced endometrial cancer.

\section{Acknowledgements}

This study is supported by a grant from the Jiangsu Provincial Department of Sciences and Technology [BS2006072 (Wu)], and by the Traditional Chinese Medicine Scientific Research Project by the Jiangsu Province Administration of Traditional Chinese Medicine [No. LZ13234 (Wu); HZ07029 (Sun)] and Project supported by the Research Foundation of Jiangsu Cancer Hospital [No. ZS201202 (Wu)].

\section{References}

1. D'Andrilli G, Bovicelli A, Paggi MG and Giordano A: New insights in endometrial carcinogenesis. J Cell Physiol 227: 2842-2846, 2012.

2. Amant F, Moerman P, Neven P, Timmerman D, Van Limbergen E and Vergote L: Endometrial cancer. Lancet 366: 491-505, 2005.

3. Hogberg T, Signorelli M, de Oliveira CF, et al: Sequential adjuvant chemotherapy and radiotherapy in endometrial cancer - results from two randomised studies. Eur J Cancer 46: 2422-2431, 2010

4. Horan TC, Zompa MA, Kim CTSKK, et al: Description of the cytotoxic effect of a novel drug abietyl-isothiocyanate on endometrial cancer cell lines. Invest New Drug 30: 1460-1470, 2012.

5. Chen YW, Lin GJ, Chia WT, Lin CK, Chuang YP and Sytwu HK: Triptolide exerts anti-tumor effect on oral cancer and KB cells in vitro and in vivo. Oral Oncol 45: 562-568, 2009.

6. Leuenroth SJ and Crews CM: Triptolide-induced transcriptional arrest is associated with changes in nuclear substructure. Cancer Res 68: 5257-5266, 2008.

7. Johnson SM, Wang XF and Evers BM: Triptolide inhibits proliferation and migration of colon cancer cells by inhibition of cell cycle regulators and cytokine receptors. J Surg Res 168: 197-205, 2011.

8. Kang DW, Lee JY, Oh DH, et al: Triptolide-induced suppression of phospholipase D expression inhibits proliferation of MDA-MB-231 breast cancer cells. Exp Mol Med 41: 678-685, 2009.

9. Li W, Liu Y, Yu Y, et al: MAPKs are not involved in triptolideinduced cell growth inhibition and apoptosis in prostate cancer cell lines with different p53 status. Planta Med 77: 27-31, 2010.

10. Matsui Y, Watanabe J, Ikegawa M, Kamoto T, Ogawa $O$ and Nishiyama H: Cancer-specific enhancement of cisplatin-induced cytotoxicity with triptolide through an interaction of inactivated glycogen synthase kinase-3 $\beta$ with p53. Oncogene 27: 4603-4614, 2008.

11. Meng HT, Zhu L, Ni WM, You LS, Jin J and Qian WB: Triptolide inhibits the proliferation of cells from lymphocytic leukemic cell lines in association with downregulation of NF- $\kappa \mathrm{B}$ activity and miR-16-1*. Acta Pharmacol Sin 32: 503-511, 2011.

12. Yu C, Zhi-hua S,Qiang W and Jian Z: Effects of tirptolide on endometrial cancer in human HEC-1B cells. Prog Obstet Gynecol 18: 282-285, 2009.

13. Jian Z, Zhi-hua S, Qiang W and Yu C: Growth inhibition effect of triptolide on human endometrial cancer cells in vivo. Acta Universitatis Medicinalis Nanjing 29: 775-778, 2009.

14. Xiao-hao H, Zhi-hua S and Qiang W: The effect of triptolide on proliferation and apoptosis signal transduction pathway$\mathrm{PI} 3 \mathrm{~K} / \mathrm{PKB}$ in human endometrial carcinoma cell line. Acta Universitatis Medicinalis Nanjing 30: 910-913, 2010.

15. Baskic D, Popovic S, Ristic P and Arsenijevic N: Analysis of cycloheximide-induced apoptosis in human leukocytes: fluorescence microscopy using annexin V/propidium iodide versus acridin orange/ethidium bromide. Cell Biol Int 30: 924-932, 2006.

16. Tong JS, Zhang QH, Huang X, et al: Icaritin causes sustained ERK1/2 activation and induces apoptosis in human endometrial cancer cells. Plos One 6: e16781, 2011 
17. Wilkins RC, Kutzner BC, Truong M, Sanchez-Dardon J and McLean JRN: Analysis of radiation-induced apoptosis in human lymphocytes: flow cytometry using Annexin V and propidium iodide versus the neutral comet assay. Cytometry 48: 14-19, 2002.

18. Yang JS, Chung JG, Wu PP, et al: Triptolide induces apoptosis in human adrenal cancer NCI-H295 cells through a mitochondrialdependent pathway. Oncol Rep 25: 551-557, 2011.

19. Cotter TG: Apoptosis and cancer: the genesis of a research field. Nat Rev Cancer 9: 501-507, 2009.

20. Sasi N, Hwang M, Jaboin J, Csiki I and Lu B: Regulated cell death pathways: new twists in modulation of BCL2 family function. Mol Cancer Ther 8: 1421-1429, 2009.

21. Xu B, Guo XX, Mathew S, Armesilla AL, Cassidy J, Darling JL and Wang WG: Triptolide simultaneously induces reactive oxygen species, inhibits NF-кB activity and sensitizes 5 -fluorouracil in colorectal cancer cell lines. Cancer Lett 291: 200-208, 2010.

22. Antonsson B: Bax and other pro-apoptotic Bcl-2 family 'killerproteins' and their victim the mitochondrion. Cell Tissue Res 306: 347-361, 2001

23. Kuhar M, Sen S and Singh N: Role of mitochondria in quercetin-enhanced chemotherapeutic response in human non-small cell lung carcinoma H-520 cells. Anticancer Res 26: 1297-1303, 2006.

24. Kim MJ, Lee TH, Kim SH,Choi YJ, Heo J and Kim YH Triptolide inactivates Akt and induces caspase-dependent death in cervical cancer cells via the mitochondrial pathway. Int J Oncol 37: 1177-1185, 2010
25. Yang M, Huang J, Pan HZ and Jin J: Triptolide overcomes dexamethasone resistance and enhanced PS-341-induced apoptosis via PI3k/Akt/NF-кB pathways in human multiple myeloma cells. Int J Mol Med 22: 489-496, 2008.

26. Cory S and Adams JM: The bcl 2 family: regulators of the cellular life-or-death switch. Nat Rev Cancer 2: 647-656, 2002.

27. Phillips PA, Dudeja V, McCarroll JA, et al: Triptolide induces pancreatic cancer cell death via inhibition of heat shock protein 70. Cancer Res 67: 9407-9416, 2007.

28. Zhu W, Hu H, Qiu P and Yan GM: Triptolide induces apoptosis in human anaplastic thyroid carcinoma cells by a p53-independent but NF-кB-related mechanism. Oncol Rep 22: 1397-1401, 2009.

29. Yao JC, Jiang ZZ, Duan WG, et al: Involvement of mitochondrial pathway in triptolide-induced cytotoxicity in human normal liver L-02 cells. Biol Pharm Bull 31: 592-597, 2008.

30. Wan CK, Wang C, Cheung HY, Yang M and Fong WF: Triptolide induces Bcl-2 cleavage and mitochondria dependent apoptosis in p53-deficient HL-60 cells. Cancer Lett 241: 31-41, 2006.

31. Carter BZ, Mak DH, Schober WD, et al: Triptolide sensitizes AML cells to TRAIL-induced apoptosis via decrease of XIAP and p53-mediated increase of DR5. Blood 111: 3742-3750, 2008.

32. Amundson SA, Myers TG and Fornace AJ Jr: Roles for p53 in growth arrest and apoptosis: putting on the brakes after genotoxic stress. Oncogene 17: 3287-3299, 1998.

33. Mano Y, Kikuchi Y, Yamamoto K, et al: Bcl-2 as a predictor of chemosensitivity and prognosis in primary epithelial ovarian cancer. Eur J Cancer 35: 1214-1219, 1999. 